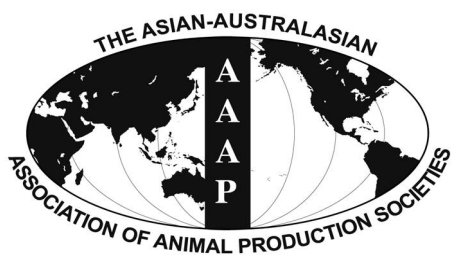

Open Access

Asian Australas. J. Anim. Sci.

Vol. 29, No. 11 : 1562-1568 November 2016

http://dx.doi.org/10.5713/ajas.15.0976

Www.ajas.info

pISSN 1011-2367 elSSN 1976-5517

\title{
Green Light-emitting Diodes Light Stimuli during Incubation Enhances Posthatch Growth without Disrupting Normal Eye Development of Broiler Embryos and Hatchlings
}

\author{
L. Zhang ${ }^{1,2}$, X. D. Zhu ${ }^{3}$, X. F. Wang ${ }^{3}$, J. L. Li ${ }^{1,2}$, F. Gao ${ }^{1,2, *}$, and G. H. Zhou ${ }^{1}$ \\ ${ }^{1}$ Jiangsu Provincial Key Laboratory of Animal Origin Food Production and Safety Guarantee, \\ College of Animal Science and Technology, Nanjing Agricultural University, Nanjing 210095, China
}

\begin{abstract}
Monochromatic green light-emitting diodes (LED) light stimuli influences the posthatch growth performance of chicks. This study was undertaken with the following objectives: i) to examine whether the green LED light stimuli induces an overheating effect by determining weight loss rate of fertile eggs during incubation period; ii) to look for the development of eyes and other primary organs at different ages of embryos and newly hatched chicks. Arbor Acres fertile broiler eggs $(n=480)$ were randomly assigned to 3 incubation groups and exposed to continuous white light, green light, or a dark environment (control) from the first day to $19 \mathrm{~d}$ of incubation. The light sourced from LED lamps with the intensity of $30 \mathrm{~lx}$ at eggshell level. The results showed that either green or white light stimuli during incubation did not significantly affect the weight loss rate of fertile eggs, hatching time, hatchability, chick embryo, or body weight (BW), the weight percentage of heart, liver, and eyes, as well as obvious systematic abnormalities in eye weight, side-toside, back-to-front, or corneal diameter from $15 \mathrm{~d}$ of embryogenesis to $6 \mathrm{~d}$ of posthatch ( $>0.05$ ). Compared with the dark condition, green light stimuli during incubation tended to increase feed intake $(\mathrm{p}=0.080)$, improved the BW gain of chicks during 0 to 6 day posthatch $(\mathrm{p}<0.05)$, and increased the percentage of pectoral muscle to the BW on 3- and 6-day-old chicks. In addition, embryos or chicks in green light had lower weight percentage of yolk retention on $19 \mathrm{~d}$ of embryogenesis and $1 \mathrm{~d}$ of posthatch in comparison to those in dark or white group $(\mathrm{p}<0.05)$. These results suggest that providing $30 \mathrm{~lx}$ green LED light stimuli during incubation has no detrimental effect on the development of eyes, heart and liver of embryos and hatchlings, but does have potential benefits in terms of enhancement of the chick growth during the early posthatch stages. In addition, the fertile broiler eggs stimulated with 30 lx green LED light during incubation does not cause an overheating effect. (Key Words: Chick Embryo, Incubation, Light Emitting Diodes, Light Stimuli)
\end{abstract}

\section{INTRODUCTION}

Light is an essential part of the physical environment and a powerful exogenous factor, which affects physiological development, behavioural processes and welfare of domestic fowls (Onbasilar et al., 2007; Parvin et

\footnotetext{
* Corresponding Author: F. Gao. Tel: +86-25-84399007, Fax: +86-25-84395314, E-mail: gaofeng0629@sina.com

2 Jiangsu Provincial Key Laboratory of Gastrointestinal Nutrition and Animal Health, Nanjing Agricultural University, Nanjing 210095, China.

${ }^{3}$ College of Science, Nanjing Agricultural University, Nanjing 210095, China.

Submitted Nov. 28, 2015; Revised Jan. 23, 2016; Accepted Feb. 15, 2016
}

al., 2014). Thus, artificial light has been widely used to modulate avian reproduction and growth rate. Over the past several decades, the light sources used in poultry husbandry are mainly incandescent or fluorescent lamps. Recently, light-emitting diodes (LED) are gradually replacing the incandescent or fluorescent lamps because they are much more energy efficient, provide adequate illumination, and improve growth performance of poultry (Rozenboim et al., 2004; Hassan et al., 2013; Kim et al., 2014; Riber et al., 2015).

Chickens have a superior nature of avian vision, and they are sensitive to different wavelengths (colors) of light because of the special extra-retinal photoreceptors in the hypothalamus or in other sites of the brain (Foster and 
Follett, 1985; Lewis and Morris, 2000). Some previous studies showed that light stimuli can be recognized by chick embryos early in their embryonic stage and has potentially positive effects on their behavioral and physiological development (Rozenboim et al., 2004; Zhang et al., 2012; 2014a). These studies suggested that green LED light stimuli during embryogenesis enhances both body and breast muscle weight at both the embryonic stage and posthatch. Stimulation with green LED light during incubation also increases the chick embryo liver weight accompanied by a high level of proliferating cell nuclear antigen (PCNA) expression and an enhanced antioxidant activity of the liver, which is mediated by melatonin levels and melatonin receptor 1c (Mel1c) expression (Wang et al., 2014).

The light stimuli have also been shown to affect eye health in chickens. Continuous lighting regimens were reported to disrupt the functional development of avian eyes during the incubation period (Aige-Gil and Murillo-Ferrol, 1992; Archer et al., 2009) or raising stage (Whitley et al., 1984; Oishi and Murakami, 1985; Li et al., 1995). Previously, we have demonstrated that stimulation with monochromatic green LED during incubation accelerates body weight (BW) and posthatch pectoral muscle growth of broilers (Zhang et al., 2012), but limited data are available for evaluating the green LED light effects on the development of eyes and other primary organs. Moreover, light-induced overheating is recognized as another potential factor that accelerates embryonic development (Rozenboim et al., 2003; 2004), but whether the growth-promoting effect of green LED light stimuli is closely related to an overheating effect is also still unknown. Therefore, the present study was undertaken with the following objectives: i) to examine whether the green LED light stimuli induces an overheating effect by determining weight loss rate of fertile eggs during incubation period; ii) to observe the development of eyes and other primary organs, including heart, liver and pectorals muscle, of embryos and newly hatched chicks.

\section{MATERIALS AND METHODS}

\section{Birds and light regimes}

Fertile eggs from 38-week-old Arbor Acres broiler breeders were purchased from Jiangsu Jinghai Poultry Industry Group Co. Ltd. (Haimen, China). All eggs ( $\mathrm{n}=$ 480) were pre-weighed individually and selected for an average weight of $60.73 \mathrm{~g}$ ranged from 56 to $63 \mathrm{~g}$. After disinfecting the eggshell with $0.1 \%$ bromogeramine solution, all eggs were randomly assigned to 3 incubation groups: i) control group in dark condition, ii) LED-white light group, and iii) LED-green light group $(560 \mathrm{~nm})$. In the light groups, the white or monochromatic green light was provided by LED lamps (Zhongshan Silsmart Optoelectronics Co., Ltd., Guangzhou, China) from the first day to $19 \mathrm{~d}$ of incubation, and then the eggs were immediately transferred to the hatchery compartment of the same incubator for hatching in the commercial dark condition. The average continuous illumination intensity at the eggshell level was $30 \mathrm{~lx}$, as measured with a digital luxmeter (Mastech MS6610, Precision Mastech Enterprises, Hong Kong, China). The incubators (HXC-5, Hengxin Incubation Equipment Co., Ltd., Nanjing, China) were maintained at a temperature of $37.6^{\circ} \mathrm{C} \pm 0.1{ }^{\circ} \mathrm{C}$ and $60 \%$ relative humidity. The eggs were compartmentalized within separate hatching trays (4 trays per treatment with 40 eggs per tray), and the hatching time and the numbers of hatched chicks were recorded every $5 \mathrm{~h}$ form $480 \mathrm{~h}$ to $510 \mathrm{~h}$. After hatch, 120 healthy chicks from each incubation group were randomly selected and placed in 8 replicates (cages) with 15 birds each. All birds were raised under LED white light (30 $1 \mathrm{x}$ at bird-head level) with a light schedule of $23 \mathrm{~h}$ light and $1 \mathrm{~h}$ dark (23L: 1D), and given free access to commercial diet and water for the first 6 experimental days. The temperature in the chicken house was set at $32^{\circ} \mathrm{C}$. The experimental procedures were approved by the Animal Care and Use Committee of Nanjing Agricultural University.

\section{Sampling and measurements}

Weight loss rate of fertile eggs: Before entering the incubator, 3 eggs from each tray were labeled and weighed (12 eggs per treatment), and these labeled eggs were reweighed on middle of embryonic day 5, 8, 11, 13, 15, 17, and 19 (E5, E8, E11, E13, E15, E17, and E19) to calculate the weight loss rate of fertile eggs.

Hatchability and growth performance: The hatchability was calculated as $(\%)=100 \times($ number of chicks hatched/ number of fertile eggs in each hatching tray), where each hatching tray was taken as a replicate. The BW gain and feed conversion ratio (FCR; feed:gain, g:g) were also calculated from the day of hatching to $6 \mathrm{~d}$ of age.

Eye measurements and organ weight: On embryonic days 15, 17, and 19 (E15, E17, and E19) and posthatch days 1,3 , and $6(\mathrm{H} 1, \mathrm{H} 3$, and $\mathrm{H} 6), 8$ embryos or chicks from each treatment were sacrificed using sodium pentobarbital anesthesias. Immediately after death, the eyes, heart, liver, yolk, and pectorals muscle were removed and weighed to calculate the weight percentage of embryos or chicks. The right eye was then used to take the eye measurements of back-to-front and side-to side diameters, and corneal diameter (Blatchford et al., 2009).

\section{Statistical analysis}

The statistical analysis of the data was performed with the SAS program (version 8.02; SAS Institute Inc., Cary, NC, USA). For hatchability and hatching weight 


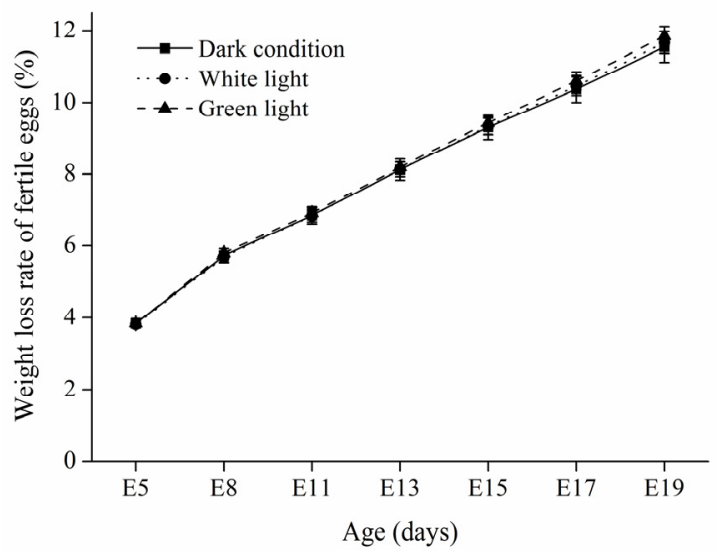

Figure 1. Weight loss rate of fertile eggs (\%) on embryonic days 5, 8, 11, 13, 15, 17, and 19 (E5, E8, E11, E13, E15, E17, and E19) incubated under light-emitting diodes-based white light, green light or dark condition. Values are presented as means \pm standard error $(n=12)$.

measurements, the individual hatching tray per treatment served as the experimental unit $(n=4)$; however, after hatch, the individual cages served as the experimental unit $(n=8)$ for statistical analysis. The normality of data was initially tested by the Shapiro-Wilk test. The statistical significance of comparisons amongst 3 experimental groups was analyzed using analysis of variance followed by Tukey's post hoc test to compare group means. Results are presented as the mean \pm standard error. Differences were considered statistically significant at $\mathrm{p}<0.05$ unless otherwise stated.

\section{RESULTS AND DISCUSSION}

Shutze et al. (1962) first reported that incandescent lighting accelerated chick embryo development and shorted incubation time compared to commercial dark incubation. Similar results were also found in White Rock chickens (Siegel et al., 1969), Rhode Island Red domestic fowls (Adam and Dimond, 1971), quails and broilers (Walter and Voitle, 1973). Overheating effect caused by incandescent lamps is recognized as a potential factor that could

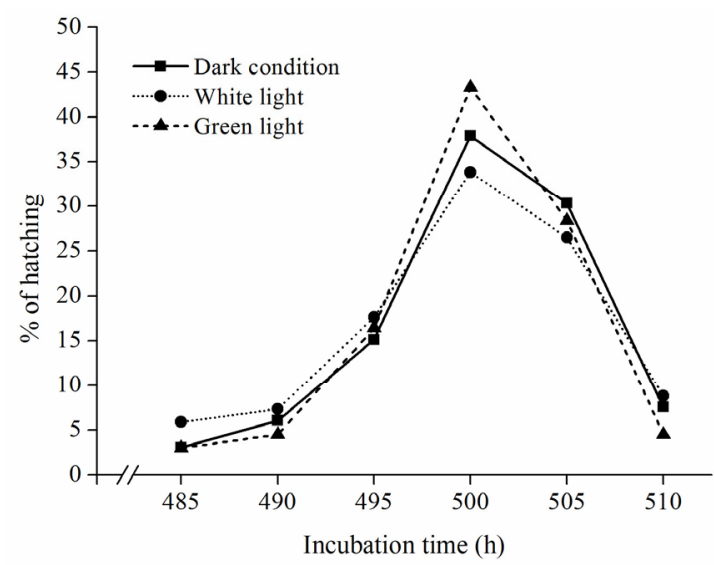

Figure 2. Percentage hatching relative to the hatching time of broiler chicks incubated under light-emitting diodes-based white light, green light or dark condition.

accelerate embryonic development (Rozenboim et al., 2003; 2004). In this study, we thus determined the weight loss rate of fertile eggs during incubation period to judge whether there was an overheating effect. The results showed that regardless of the experimental treatment effect, the weight loss rate of fertile eggs increased with the extension of incubation time from E5 (3.83\%) to E19 (11.67\%) (Figure 1). However, no significant difference was observed in weight loss rate of fertile chick eggs from E5 to E19 days of age amongst 3 experimental groups ( $>0.05$; Figure 1), implying that an overheating effect caused by $30 \mathrm{~lx}$ LED light was unlikely.

Shafey and Al-mohsen (2002) reported that 20-watt green fluorescent light stimulation of meat breeder eggs during the first 18 days of incubation reduced incubation period and increased embryonic growth and hatchability. On the contrary, our present study showed that both green and white LED light did not affect hatching time ( $\mathrm{p}>0.05$; Figure 2) or hatchability ( $p>0.05$; Table 1$)$, which is in accordance with previous studies on broilers (Rozenboim et al., 2004; Zhang et al., 2014a). The difference in results observed between these studies probably was probably due to an overheating effect caused by 20 -watt green fluorescent

Table 1. Effects of light-emitting diodes-based light stimuli during incubation on hatching weight, hatchability and chick growth performance from o to 6 days posthatch

\begin{tabular}{|c|c|c|c|c|c|}
\hline$\overline{\text { Items }}$ & Dark condition & White light & Green light & SEM & p-value \\
\hline$\overline{\text { Egg weight }^{1}(\mathrm{~g})}$ & 60.70 & 60.72 & 60.81 & 0.071 & 0.941 \\
\hline Hatchability $^{1}(\%)$ & 91.67 & 93.15 & 91.78 & 1.443 & 0.852 \\
\hline Hatching weight $^{1}(\mathrm{~g})$ & 45.34 & 45.96 & 46.11 & 0.308 & 0.408 \\
\hline \multicolumn{6}{|l|}{0 to 6 days } \\
\hline BW gain² (g/bird) & $62.91^{\mathrm{b}}$ & $64.06^{\mathrm{ab}}$ & $67.04^{\mathrm{a}}$ & 0.752 & 0.043 \\
\hline Feed intake ${ }^{2}(\mathrm{~g} / \mathrm{bird})$ & 90.32 & 91.81 & 96.03 & 1. 601 & 0.080 \\
\hline FCR $^{2}$ (feed:gain, g:g) & 1.436 & 1.433 & 1.432 & 0.017 & 0.933 \\
\hline
\end{tabular}

LED, light-emitting diodes; SEM, standard error of the mean; FCR, feed conversion ratio (feed:gain, g:g).

${ }^{1}$ Values are presented as mean $\pm \operatorname{SEM}(n=4)$, since the individual hatching tray per treatment $(n=4)$ were served as the experimental unit.

${ }^{2}$ Values are presented as mean $\pm \operatorname{SEM}(n=8)$, since the individual cages $(n=8)$ were served as the experimental unit after hatch.

${ }^{\mathrm{a}, \mathrm{b}}$ Means in the same row with different superscripts differ significantly $(\mathrm{p}<0.05)$. 
lamps used by Shafey and Al-mohsen (2002). The first week posthatch is critical for chicks in terms of evaluation of growth potential, including muscle satellite cell mitotic activity, digestive organ development, and BW gain (Moore et al., 2005; Zhang et al., 2014a; Lilburn and Loeffler, 2015). In our study, compared with the control group, green LED light stimuli during incubation tended to increase feed intake ( $\mathrm{p}=0.080)$, and improve the BW gain of chicks from o to 6 days posthatch $(\mathrm{p}<0.05$; Table 1$)$, which once again demonstrated that green LED light has positive effects on chick growth during the early posthatch stages. More importantly, the power consumption of green LED lamps was approximately $2 \mathrm{kWh}(4$ watts $\times 24 \mathrm{~h} / \mathrm{d} \times 19 \mathrm{~d})$, which only marginally increased the cost compared to dark incubation. According to some published reports, the possible mechanisms of the growth-promoting effect in broilers of green LED light stimuli during incubation can be summarized as follows i) green light stimuli enhances the proliferation and differentiation of satellite cells in late embryonic stages, and subsequent muscle hypertrophy (Halevy et al., 2006; Zhang et al., 2014a); ii) green light increases melatonin secretion by influencing circadian rhythm of melatonin biosynthesis in the embryo pineal gland, which increased the proliferative activity and the antioxidant status of hepatocytes (Piesiewicz et al., 2012; Wang et al., 2014); and iii) the higher secretion of somatotropic axis hormones (growth hormone [GH] and insulin-like growth factor-I [IGF-I]) may be another important contributor to chicken growth promoted by green light stimuli during embryogenesis (Zhang et al., 2014b).

The eyes of chickens are beginning to develop after 48 hour incubation, and the most useful criteria to determine their development can be obtained after 12th incubation days (Hamburger and Hamilton, 1951). Exogenous light can penetrate the eggshell and be recognized by eyes of chick embryos, and then affects the function of their lightsensitive pineal gland in the late incubation stage (Aige-Gil and Murillo-Ferrol, 1992; Zeman et al., 1992). However, light stimuli is also a source of corresponding risks to the development of avian eyes. Whitley et al. (1984) found that chickens raised from 2 weeks of age in continuous fluorescent light $(1,044 \mathrm{~lx})$ displayed abnormal buphthalmic eyes (in which corneal diameters and axial globe lengths the eyes become enlarged) and even blindness. Direct exposed to a source of white light during incubation also may cause a number of teratological effects in chickens such as higher mortality, lower eye weight, and the increase in number and size of the intracytoplasmic lipid droplets of the follicular pinealocytes (Aige-Gil and Murillo-Ferrol, 1992; Archer et al., 2009). However, the present study showed that providing $30 \mathrm{~lx}$ green or white LED light during incubation did not affect any of measured eye parameters, including weight, side-to-side, back-to-front or corneal diameter $(\mathrm{p}>0.05 ;$ Figure 3$)$. In addition, no
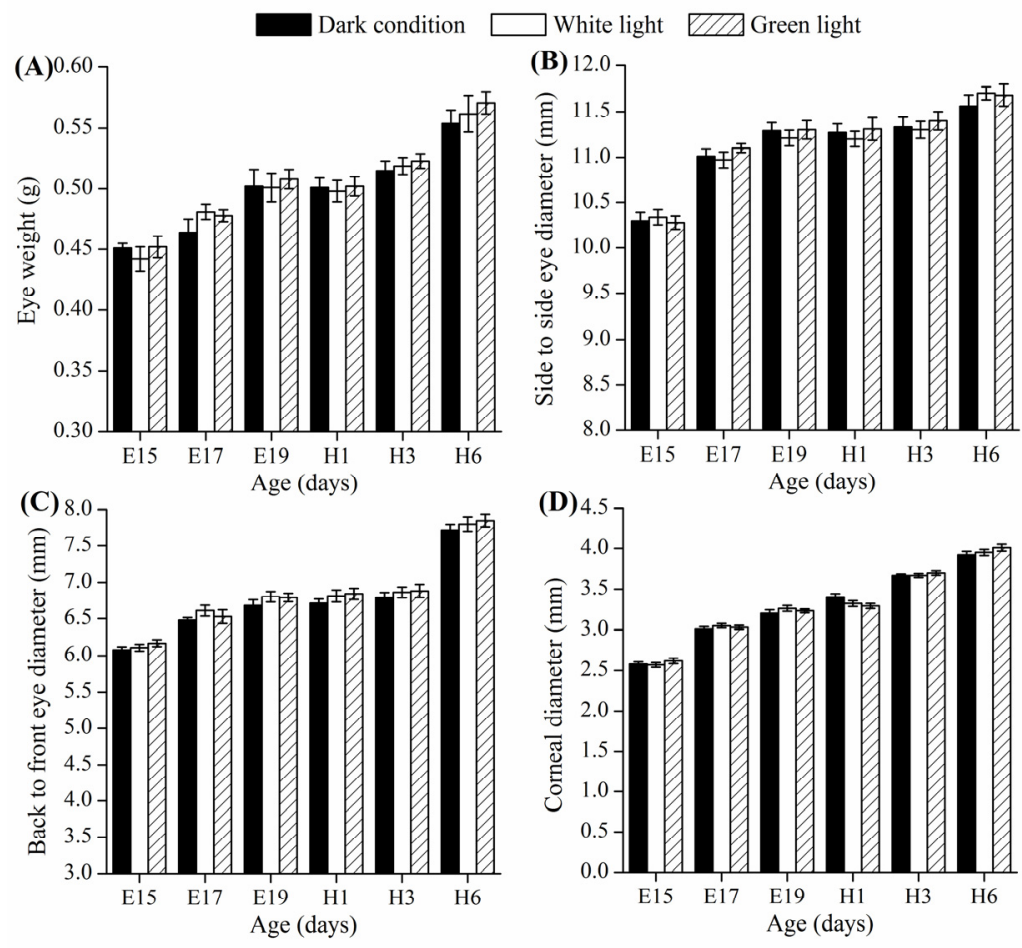

Figure 3. (A) Eye weight, (B) side to side or (C) back to front eye diameters, and (D) corneal diameter of chick embryos on embryonic days 15, 17, and 19 (E15, E17, and E19) and newly hatched chicks on posthatch days 1, 3, and 6 (H1, H3, and H6) incubated under lightemitting diodes-based white light, green light or dark condition. Values are presented as means \pm standard error $(\mathrm{n}=8)$. 
significant difference in embryo weight, chick BW, the weight percentage of eye, heart and liver to embryo weight or chick BW from days E15 to H6 ( $>0.05$; Figure 4) was observed among all experimental groups. However, $30 \mathrm{~lx}$ green LED light stimuli improved the weight percentage of pectoral muscle on day $\mathrm{H} 3$ and $\mathrm{H} 6$, which is a little sooner than the results of Zhang et al. (2014a), who reported that $15 \mathrm{~lx}$ green light stimulation during incubation accelerated BW and pectoral muscle growth starting from $7 \mathrm{~d}$ of age posthatch. This difference was observed probably because the light intensity in the present study (30 1x) was higher than that used by Zhang et al. (2014a). In addition, embryos or chicks in green light group had lower weight percentage of yolk retention on days E19 and $\mathrm{H} 1$ in comparison to those in dark or white groups (Figure 4), mainly because the embryos in green light group developed faster, resulting in higher nutrient consumption from yolk.

In conclusion, providing $30 \mathrm{~lx}$ green LED light stimuli during incubation has no detrimental effect on the development of eyes, heart and liver of embryos and hatchlings, but does have potential benefits in terms of enhancement of the chick growth during the early posthatch
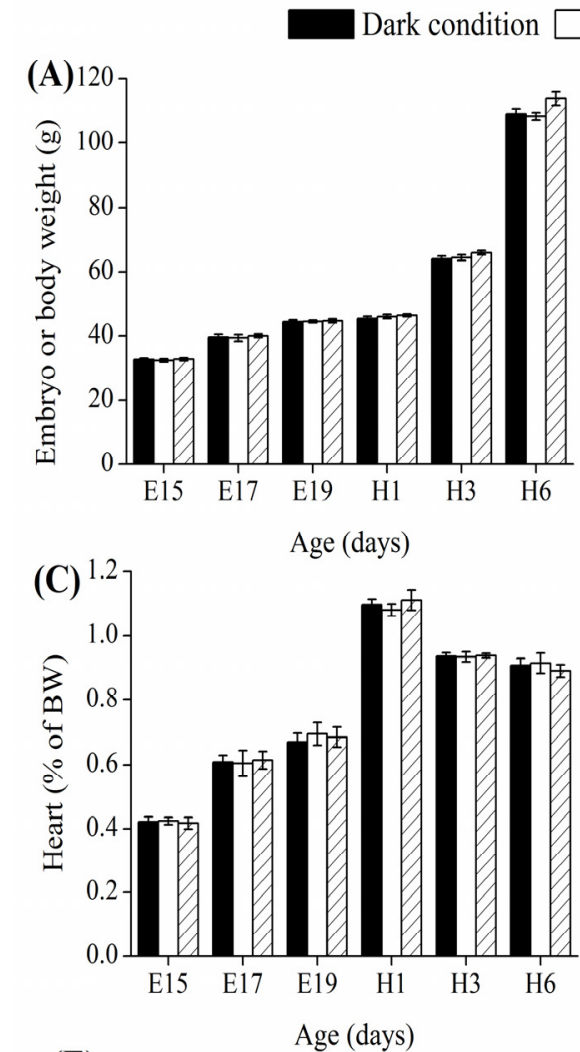

White light $\square]$ Green light
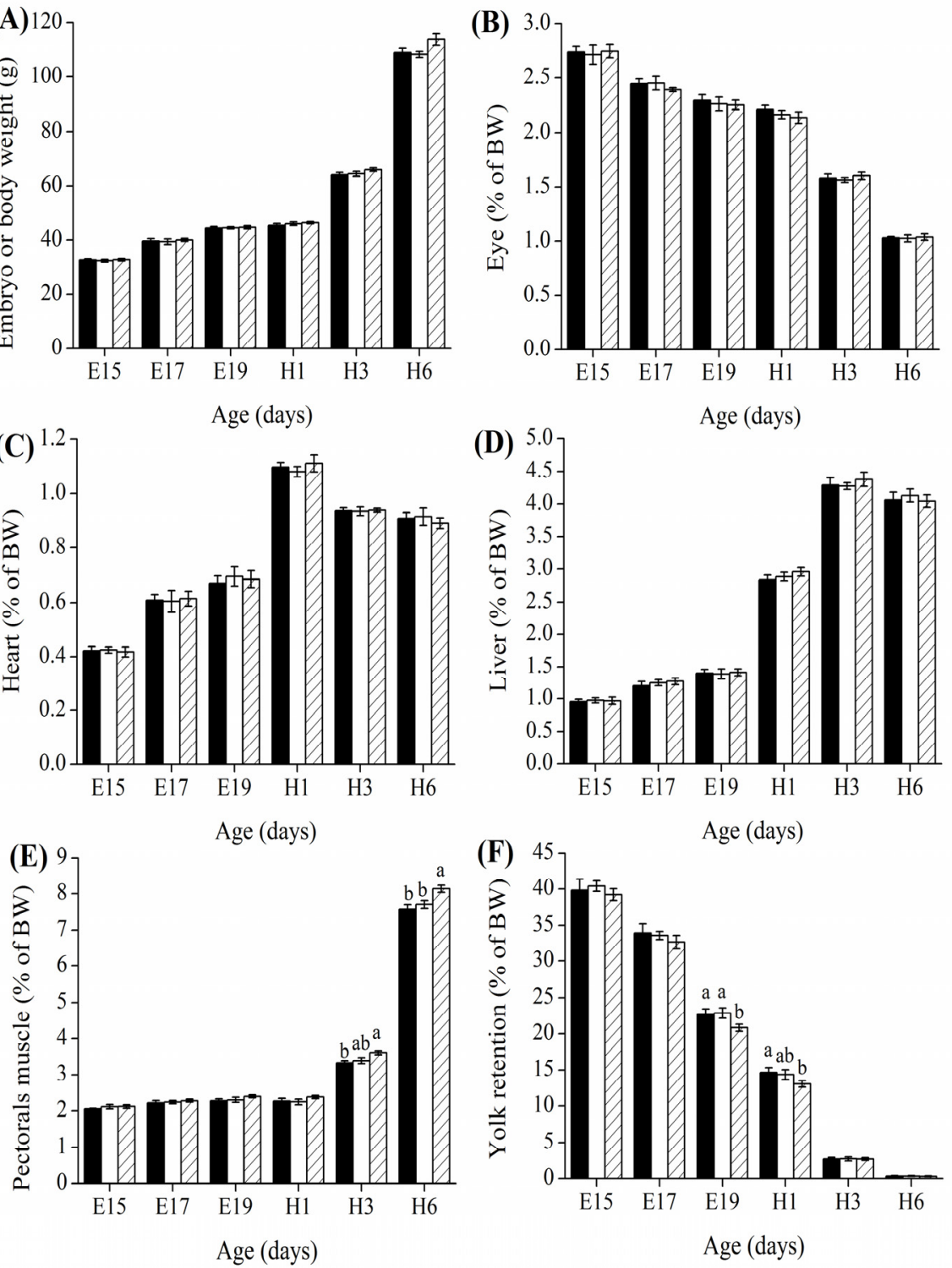

Figure 4. Chick (A) embryo (with yolk body mass) or body weight, (B) eye, (C) heart, (D) liver, (E) pectorals muscle, and (F) yolk retention weight percentage of body weight (BW) of chick embryos on embryonic days 15, 17, and 19 (E15, E17, and E19) and newly hatched chicks on posthatch days 1, 3, and 6 (H1, H3, and H6) incubated under light-emitting diodes-based white light, green light or dark condition. Values are presented as means \pm standard error $(n=8)$. Bars with different letters indicate significant differences between treatments at the same age $(\mathrm{p}<0.05)$. 
stages. In addition, $30 \mathrm{~lx}$ green LED light stimulation during incubation does not cause an overheating effect on fertile broiler eggs.

\section{CONFLICT OF INTEREST}

We certify that there is no conflict of interest with any financial organization regarding the material discussed in the manuscript.

\section{ACKNOWLEDGMENTS}

This research was funded by the Research Fund for the Doctoral Program of Higher Education of China (No. 20130097120051).

\section{REFERENCES}

Adam, J. H. and S. J. Dimond. 1971. Influence of light on the time of hatching in the domestic chick. Anim. Behav. 19:226-229.

Aige-Gil, V. and N. Murillo-Ferrol. 1992. Effects of white light on the pineal gland of the chick embyro. Histol. Histopathol. 7:16.

Archer, G. S., H. L. Shivaprasad, and J. A. Mench. 2009. Effect of providing light during incubation on the health, productivity, and behavior of broiler chickens. Poult. Sci. 88:29-37.

Blatchford, R. A., K. C. Klasing, H. L. Shivaprasad, P. S. Wakenell, G. S. Archer, and J. A. Mench. 2009. The effect of light intensity on the behavior, eye and leg health, and immune function of broiler chickens. Poult. Sci. 88:20-28.

Foster, R. G. and B. K. Follett. 1985. The involvement of a rhodopsin-like photopigment in the photoperiodic response of the Japanese quail. J. Comp. Physiol. A. 157:519-528.

Halevy, O., Y. Piestun, I. Rozenboim, and Z. Yablonka-Reuveni. 2006. In ovo exposure to monochromatic green light promotes skeletal muscle cell proliferation and affects myofiber growth in posthatch chicks. Am. J. Physiol. Regul. Integr. Comp. Physiol. 290: R1062-R1070.

Hamburger, V. and H. L. Hamilton. 1951. A series of normal stages in the development of the chick embryo. J. Morph. 88:49-92.

Hassan, M. R., S. Sultana, H. S. Choe, and K. S. Ryu. 2013. Effect of monochromatic and combined light colour on performance, blood parameters, ovarian morphology and reproductive hormones in laying hens. Ital. J. Anim. Sci. 12:e56.

Kim, N., S. R. Lee, and S. J. Lee. 2014. Effects of light color on energy expenditure and behavior in broiler chickens. Asian Australas. J. Anim. Sci. 27:1044-1049.

Lewis, P. D. and T. R. Morris. 2000. Poultry and colored lights. Worlds Poult. Sci. J. 56:189-207.

Li, T., D. Troilo, A. Glasser, and H. C. Howland. 1995. Constant light produces severe corneal flattening and hyperopia in chickens. Vision Res. 35:1203-1209.

Lilburn, M. S. and S. Loeffler. 2015. Early intestinal growth and development in poultry. Poult. Sci. 94:1569-1576.

Moore, D. T., P. R. Ferket, and P. E. Mozdziak. 2005. Muscle development in the late embryonic and early post-hatch poult. Int. J. Poult. Sci. 4:138-142.

Oishi, T. and N. Murakami. 1985. Effects of duration and intensity of illumination on several parameters of the chick eye. Comp. Biochem. Physiol. A Physiol. 81:319-323.

Onbasilar, E. E., H. Erol, Z. Cantekin, and U. Kaya. 2007. Influence of intermittent lighting on broiler performance, incidence of tibial dyschondroplasia, tonic immobility, some blood parameters and antibody production. Asian Australas. J. Anim. Sci. 20:550-555.

Parvin, R., M. M. H. Mushtaq, M. J. Kim, and H. C. Choi. 2014. Light emitting diode (LED) as a source of monochromatic light: a novel lighting approach for behaviour, physiology and welfare of poultry. Worlds Poult. Sci. J. 70:543-556.

Piesiewicz, A., U. Kedzierska, E. Podobas, I. Adamska, K. Zuzewicz, and P. M. Majewski. 2012. Season-dependent postembryonic maturation of the diurnal rhythm of melatonin biosynthesis in the chicken pineal gland. Chronobiol. Int. 29:1227-1238.

Riber, A. B. 2015. Effects of color of light on preferences, performance, and welfare in broilers. Poult. Sci. 94:1767-1775.

Rozenboim, I., R. Huisinga, O. Halevy, and M. E. El Halawani. 2003. Effect of embryonic photo stimulation on the posthatch growth of turkey poults. Poult. Sci. 82:1181-1187.

Rozenboim, I., Y. Piestun, N. Mobarkey, M. Barak, A. Hoyzman, and O. Halevy. 2004. Monochromatic light stimuli during embryogenesis enhance embryo development and posthatch growth. Poult. Sci. 83:1413-1419.

SAS (Statistical Analysis System) Institute. 2001. SAS User's Guide. Version 8.02. SAS Institute Inc., Cary, NC, USA.

Shafey, T. M. and T. H. Al-mohsen. 2002. Embryonic growth, hatching time and hatchability performance of meat breeder eggs incubated under continuous green light. Asian Australas. J. Anim. Sci. 15:1702-1707.

Shutze, J. V., J. K. Lauber, M. Kato, and W. O. Wilson. 1962. Influence of incandescent and coloured light on chicken embryos during incubation. Nature 196:594-595.

Siegel, P. B., S. T. Isakson, F. N. Coleman, and B. J. Huffman. 1969. Photoacceleration of development in chick embryo. Comp. Biochem. Physiol. 28:753-758.

Walter, J. H. and R. A. Voitle. 1973. Effects of photoperiod during incubation on embryonic and post-embryonic development of quail and chickens. Br. Poult. Sci. 14:533-540.

Wang, T., Z. Wang, J. Cao, Y. Dong, and Y. Chen. 2014. Monochromatic light affects the development of chick embryo liver via an anti-oxidation pathway involving melatonin and the melatonin receptor Mel1c. Can. J. Anim. Sci. 94:391-400.

Whitley, R. D., R. A. Albert, G. R. McDaniel, R. N. Brewer, E. C. Mora, and R. A. Henderson. 1984. Photo-induced buphthalmic avian eyes. 1. continuous fluorescent light. Poult. Sci. 63:1537-1542.

Zeman, M., E. Gwinner, and E. Somogyiova. 1992. Development of melatonin rhythm in the pineal-gland and eyes of chickembryo. Experientia 48:765-768.

Zhang, L., H. J. Zhang, X. Qiao, H. Y. Yue, S. G. Wu, J. H. Yao, and G. H. Qi. 2012. Effect of monochromatic light stimuli during embryogenesis on muscular growth, chemical composition, and meat quality of breast muscle in male 
broilers. Poult. Sci. 91:1026-1031.

Zhang, L., H. J. Zhang, J. Wang, S. G. Wu, X. Qiao, H. Y. Yue, J.

H. Yao, and G. H. Qi. 2014a. Stimulation with monochromatic green light during incubation alters satellite cell mitotic activity and gene expression in relation to embryonic and posthatch muscle growth of broiler chickens. Animal 8:86-93.
Zhang, L., S. Wu, J. Wang, X. Qiao, H. Yue, J. Yao, H. Zhang, and G. Qi. 2014b. Changes of plasma growth hormone, insulin-like growth factors-I, thyroid hormones, and testosterone concentrations in embryos and broiler chickens incubated under monochromatic green light. Ital. J. Anim. Sci. 13:530535. 